\title{
The different forms of the left brachiocephalic vein visualised during cardiovascular implantable electronic device implantation procedures
}

\author{
R. Steckiewicz'1 ${ }^{1}$, P. Stolarz², B. Lange \\ ${ }^{1}$ Department of Cardiology, Central University Hospital in Warsaw, Poland \\ ${ }^{2} 1^{\text {st }}$ Department of Cardiology, Medical University of Warsaw, Poland \\ [Received: 6 October 2021; Accepted: 10 November 2021; Early publication date: 26 November 2021]
}

Background: Among the factors ensuring successful completion of such minimally invasive procedures as cardiac implantable electronic device (CIED) implantation and central venous catheter (CVC) placement are the morphometry and topography of the vessels used for cardiac lead or catheter advancement. Venous access through the left clavipectoral triangle makes use of the left brachiocephalic vein $(B C V)$. The purpose of this study was to present the radiology images of various individual forms of this vessel observed during CIED implantation procedures.

Materials and methods: Our analysis included 100 venography recordings illustrating the left $B C V$, obtained during de novo CIED implantation procedures. We assessed the mediastinal course of the left $B C V$, with its natural angles, including angle $\alpha$ (in the middle section of the vessel) and the two angles created by the left $B C V$ and the left subclavian vein (angle $\beta$ ) and the left $B C V$ and the superior vena cava (angle $\gamma$ ).

Results: The mean values of angle $\alpha$ tended to be higher (approximately $141^{\circ}$ ) than those of the two remaining angles ( $\gamma$ and $\beta$ ), which were comparable at $123^{\circ}$ and $127^{\circ}$, respectively. An increase in mean angle $\alpha$ values were accompanied by increased mean angle $\gamma$ and $\beta$ values ( $p=0.05$ ), with only $5 \%$ of $\beta$ and $\gamma$ angles, in total, having values close to those of a right angle $\left(90 \pm 10^{\circ}\right)$.

Conclusions: Individual variability of left BCV topography and morphology comes from developmental formation of the physiological angle in the middle section of this mediastinal vessel's course. The presence of near-right angles along the course of left BCV may potentially result in injuries to the vessel during intravascular procedures. (Folia Morphol 2023; 82, 1: 127-136)

Key words: left brachiocephalic vein, anatomic variation, venography, cardiac implantable electronic device (CIED), central venous catheter (CVC), risk factors, anatomical factors 

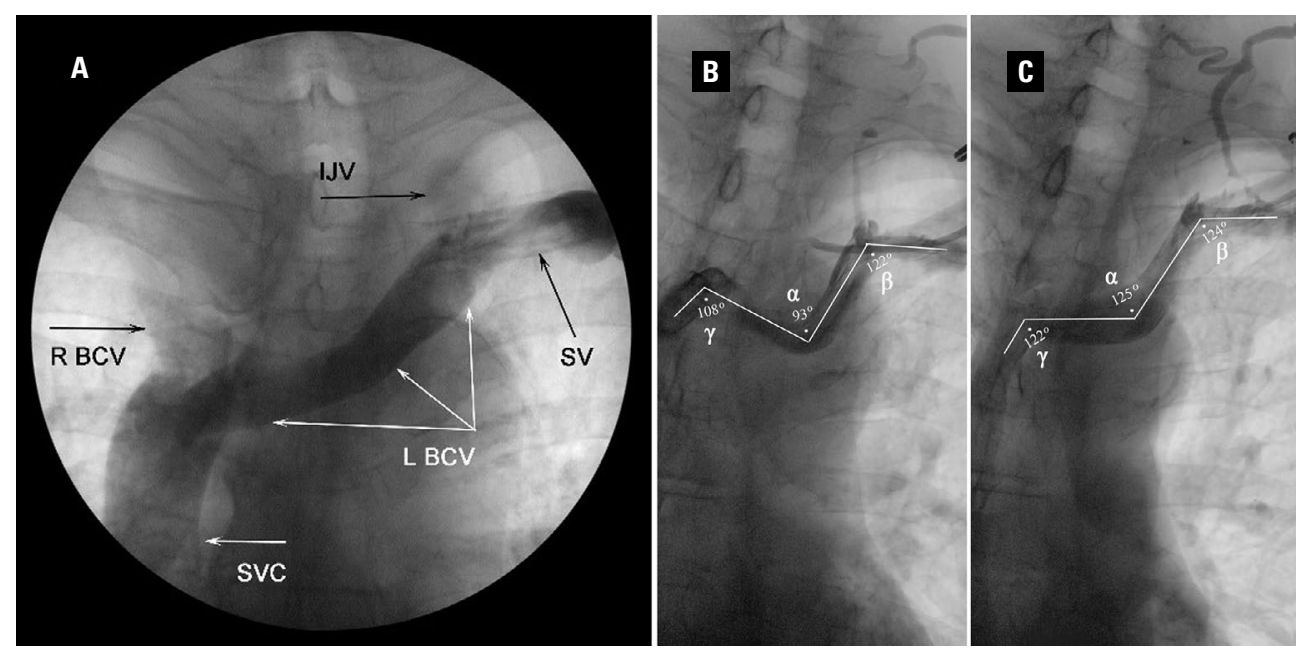

Figure 1. A-C. Radiographic images of the left brachiocephalic vein (BCV) lumen visualised with a contrast medium with respect to the contours of upper mediastinal organs (e.g. the aortic arch, trachea) and bone structures of the chest; B-C. Respiratory phase-dependent variability in the shape of left BCV angulations (posteroanterior view, supine position); B. Expiratory phase; C. Inspiratory phase: angles $\alpha, \beta$, and $\gamma$ increased by $32^{\circ}, 2^{\circ}$, and $14^{\circ}$, respectively; IJV — internal jugular vein; SV — subclavian vein; SVC — superior vena cava.

\section{INTRODUCTION}

Performing minimally invasive transvenous procedures, such as cardiac implantable electronic device (CIED) implantation, facilitates making observations as to the individual differences in vessel layout. These differences can be due to either normal anatomical variations or developmental anomalies [3, 12, 15, 19].

Minimally invasive CIED implantation or central venous catheter (CVC) placement procedures, which make use of mediastinal systemic veins, depend to a large extent on favourable morphometry and topography of the brachiocephalic veins (BCVs), also known as the innominate veins. When the venous system is accessed via the left clavipectoral triangle, the vein used for catheter or cardiac lead advancement within the middle mediastinum is the left BCV — formed by the left jugular vein and the left subclavian vein (SV) merging - which subsequently drains to the superior vena cava (SVC) (Fig. 1A).

Left BCV topography, which depends on the nature and size of the natural anatomical angulations along the course of the vessel, may affect the course of CIED implantation or CVC placement procedures $[5,18]$. These natural angulations include: the angle at the middle segment of the left BCV, where its wall is anterior and adjacent to the arteries branching off the aortic arch (angle $\alpha$, Fig. 1), and two other evaluated angles: one located at the site where the left SV continues into the left BCV (angle $\beta$, Fig. 1B, C) and the other located at the site of drainage of the left BCV into the SVC (angle $\gamma$, Fig. 1B, C).
The extent of individual variations and the spatial relations of the left BCV to adjacent anatomical structures may contribute to vessel injury during CIED implantation or CVC placement procedures even in the case of a physiological course of the left BCV $[11,13,14,16]$. There may be direct mechanical injury to some or all the layers of the venous wall, with additional damage to the adjacent arteries in more severe cases; there may also be long-term complications in the form of post-thrombotic lesions at the site of vascular injury $[2,9,10,17]$.

The risk of vascular injury is particularly high in the case of anatomical variations of considerably unusual morphometry and topography. With the angulations along the left BCV course seemingly constituting the most vulnerable sites [5, 18, 22].

The fact that left BCV variations mentioned above are typically asymptomatic means that they are usually detected incidentally during an intravascular procedure [7]. The purpose of this study was to present radiographic data on different forms of the left BCV and the analysis of the types and rates of the individual forms of the vein encountered during CIED implantation procedures over a 6-year period.

\section{MATERIALS AND METHODS}

We retrospectively analysed the venography images obtained during de novo CIED implantation procedures performed during the period of 2014-2019. Only the images that definitively and clearly showed the left BCV 

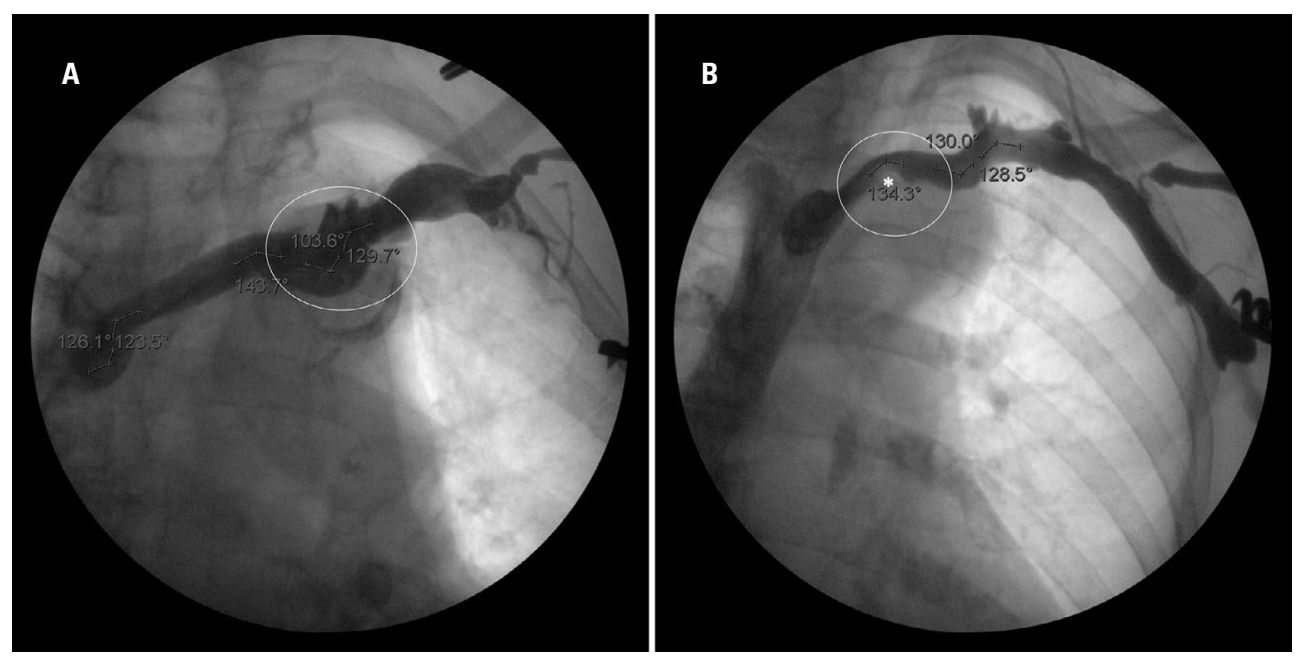

Figure 2. A, B. Radiographic examples of left brachiocephalic vein anomalies (white ovals); A. An anomaly with respect to the normal course of the vein at the place where the left subclavian vein drains into the left brachiocephalic vein (the potential location of angle $\beta$ ), most likely due to an additional angulation of the vein in a sagittal plane; $\mathbf{B}$. Locally altered morphometry of the vein due to its partial compression via the brachiocephalic trunk (white asterisk).

course, including the entire length of its lumen and the junctions with the left SV and the SVC visualised via a contrast medium, were considered in the analysis (Fig. 1)

The cases where left BCV lumen morphometry had been difficult to interpret or the angles to measure due to such factors as developmental anomalies of the vessel, poorly contrasted images, past cardiothoracic surgeries, or an atypical left $B C V$ course, were excluded from analysis (Fig. 2A, B).

Following the exclusion of some images for the reasons mentioned above, 100 venographic images illustrating the evaluated angulations and the course of the left BCV were ultimately analysed. The images selected for this paper were ones that most clearly show the relevant left BCV forms and angulations. All implantations procedures were performed by operators from the same group of personnel. This limited the effects of potentially diverse levels of operator experience and varying venography techniques on the extent of vein visualisation and the later analysis of left BCV forms.

During the analysed procedures, the cardiac leads were introduced into the cardiovascular system via the veins of the left clavipectoral triangle. The indications for contrast venography were intraoperative situations that required an immediate elucidation of the cause of difficulties in lead advancement.

Selective venographies were used, with the contrast agent administered directly at the site of cephalic vein cutdown or axillary or subclavian vein puncture. This helped effectively visualise the lumina of the veins of the upper mediastinum, while simultaneously limiting the volume of contrast in comparison to that administered into peripheral veins of the arm. The intravascular flow of contrast was visualised anteroposteriorly via pulsed fluoroscopy at 12 frames per second as well as captured on individual frames.

The evaluated angles were measured at the points where the straight lines drawn along the middle of the contrasted lumina of adjacent venous segments intersected (Fig. 1B, C). In order to ensure data comparability, all venous angle measurements were made on the images obtained during the expiratory phase. We believe that the changes to the left BCV course, including the angles, taking place during that phase may increase the risk of a potential local injury to the vessel during CIED implantation or CVC placement.

Our statistical analysis used: numerical variables in the form of mean values, standard deviations, statistical significance ( $p$-values), tests for normality: the Shapiro-Wilk W test, Kolmogorov-Smirnov D test, Levene's test for homogeneity of variance, Pearson correlation coefficients. The $p$-value was $5 \%$ if not stated otherwise.

This study had been approved by the Institutional Review Board.

\section{RESULTS}

Out of the 2,112 de novo CIED implantation procedures performed over the evaluated period, we initially selected the 391 that had required the use of venography to visualise the vessels through which the cardiac lead was being advanced. Due to our 


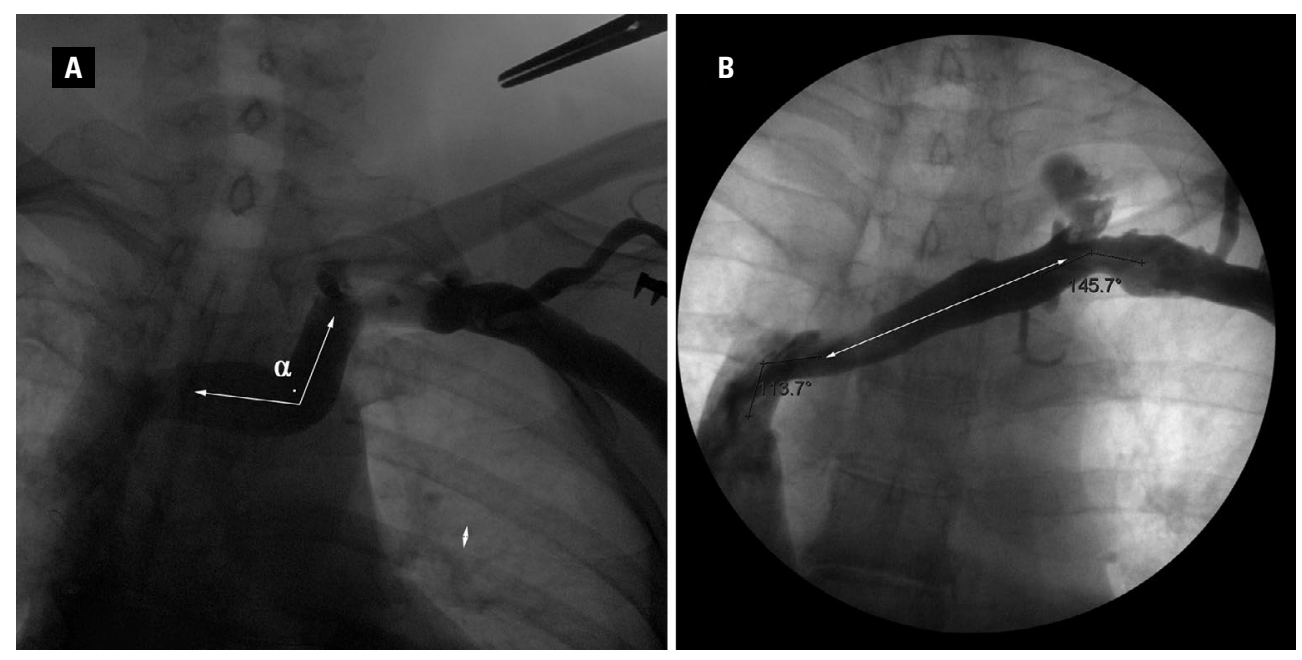

Figure 3. A, B. Interindividual physiological variations of the forms and values of angle $\alpha$ along the left brachiocephalic vein (expiratory phase); A. Vein form with angle $\alpha=100^{\circ}$; B. Vein form with no visible angulation (angle $\alpha=180^{\circ}$ ).

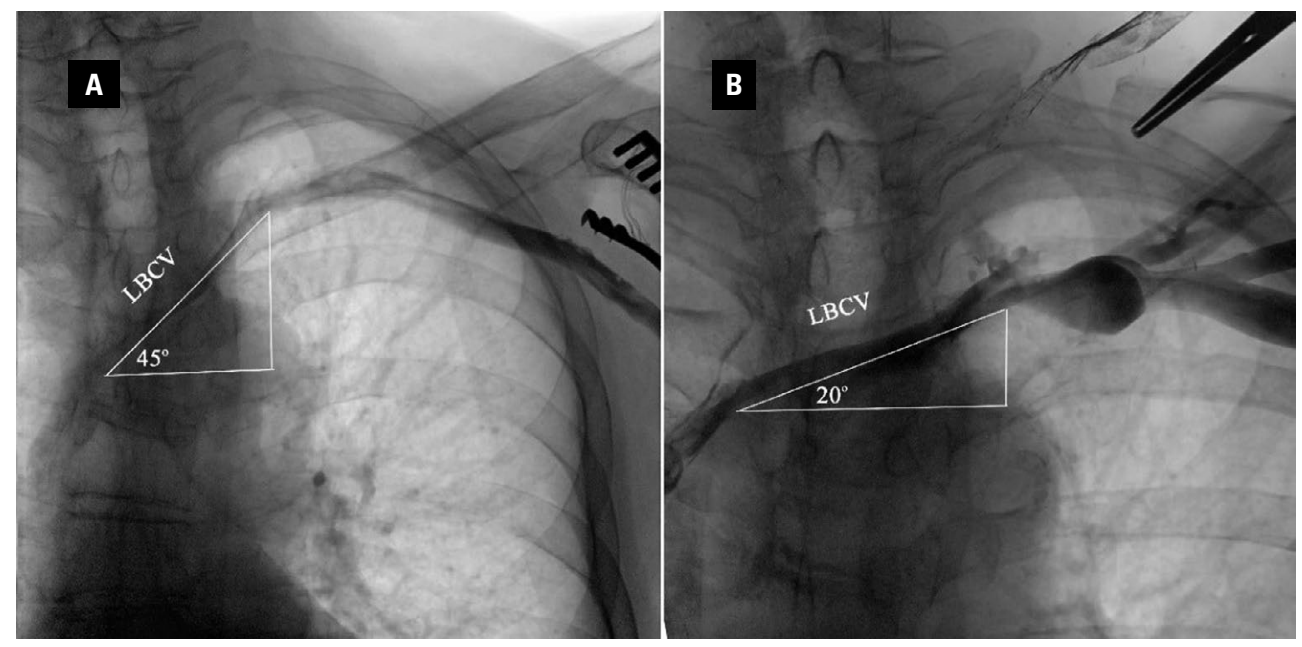

Figure 4. A, B. The extent of interpersonal variations in the slope of the left brachiocephalic vein with respect to the transverse plane (posteroanterior view).

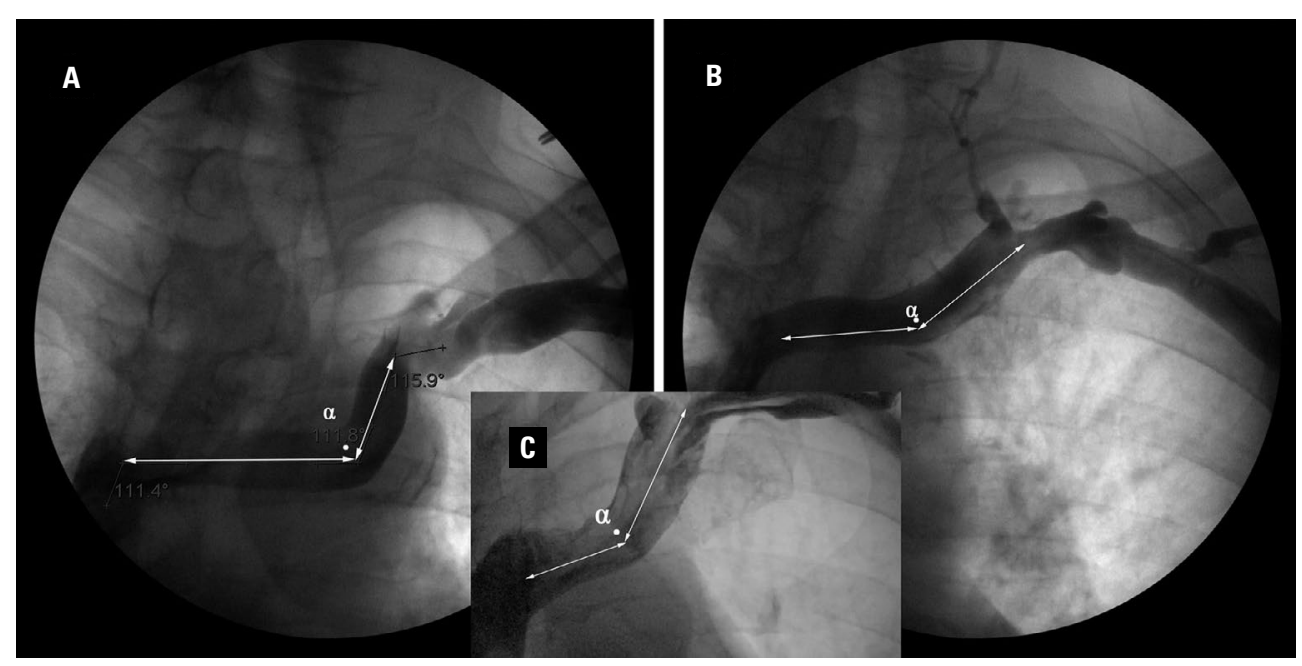

Figure 5. A, B. The location and characteristics of the vertex of left brachiocephalic vein angulation $\alpha$ within the mediastinum, with the angle-forming upstream (oblique) and downstream (horizontal) segments of the vein marked with double-headed arrows; $\mathbf{A}$. The downstream segment longer than the upstream segment; B. The downstream segment equal to the upstream segment; C. The downstream segment shorter than the upstream segment. 


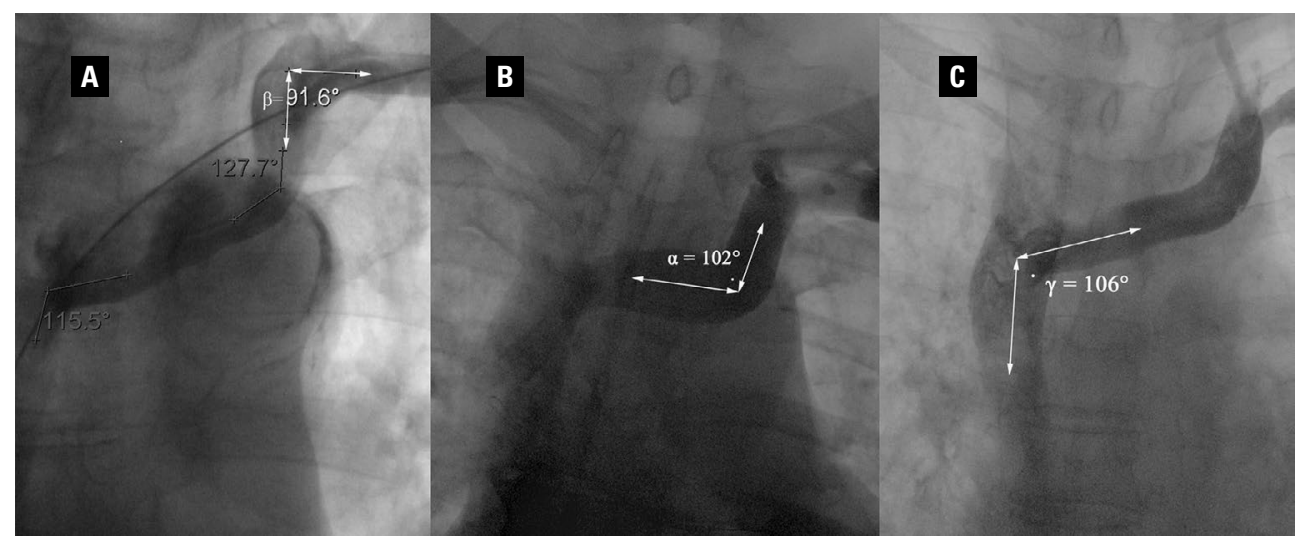

Figure 6. A-C. Examples of the three analysed left brachiocephalic vein angulations that might increase the risk of injury to the vessel during transvenous procedures.

objectives, which required an unequivocal interpretation of the obtained left BCV images, the number of recordings selected for analysis was ultimately pared down to 100. The selection criteria included good visualisation of the entire lumen of the vein's course through the mediastinum, including the SV drainage point and the left BCV drainage point to the SVC, in order to allow the evaluated angles $(\alpha, \beta$, and $\gamma)$ to be measured (Fig. 1B, C).

In the cases with no discernible angulation in the left BCV and the vein's oblique course through the mediastinum (Fig. 3B), the vein's slope with respect to the transverse plane ranged from $20^{\circ}$ to $45^{\circ}$ (Fig. 4A, B).

The position of the left $B C V$ angle vertex along the vein's length helped distinguish three main types of vein forms in the evaluated material: the predominant form (shown in Fig. 5A) present in $80 \%$ of cases, a less common form (shown in Fig. 5B) present in 15\% of cases, and the least common form (shown in Fig. 5C) present in only $5 \%$ of cases.

Considered collectively, angles $\beta$ and $\gamma$ of approximately $90^{\circ} \pm 10^{\circ}$ were observed in $5 \%$ (Figs. $1 \mathrm{~B}$; $6 \mathrm{~A}-\mathrm{C})$.

\section{Statistical analysis of the results}

\section{Basic descriptive statistics}

Description: The evaluated angles differ in terms of their mean values, with angle $\alpha$ characterised by the highest value of about $140.6^{\circ}$, and the remaining two angle types characterised by comparable lower values (angles $\gamma$ of approximately $122.8^{\circ}$ and angles $\beta$ of $126.7^{\circ}$ ).

The measurements of all angles show similar standard deviations (a range of 15.4-16.1) (Fig. 7).

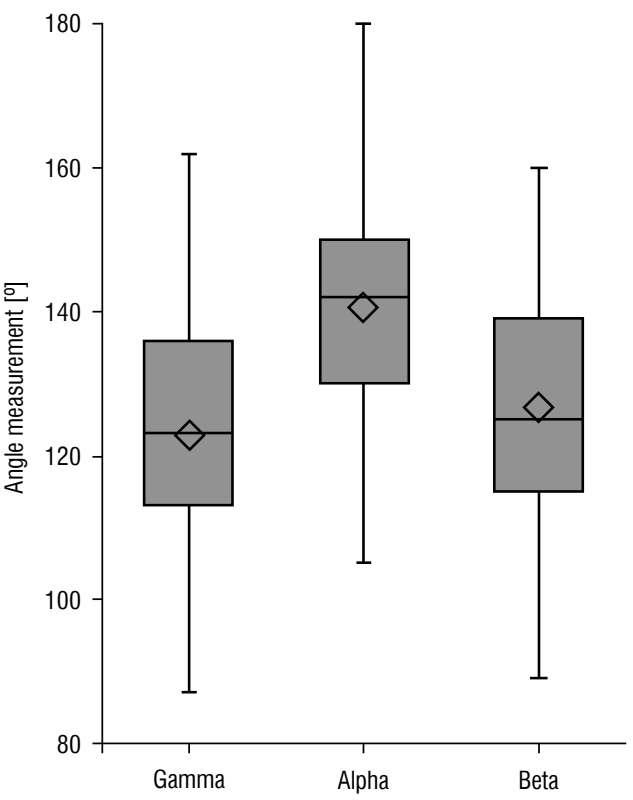

Figure 7. A box-and-whisker plot of the measured values for each angle. The bottom edge of each box represents the first quartile; the line across the box represents the median value; the top edge of each box represents the third quartile. The diamonds mark the mean value, and the whiskers show the minimum and maximum values.

\section{Histogram analysis}

Description: The histograms show the proportion of angle values falling within the defined value ranges (Fig. 8A-C).

Gamma angle: The most commonly measured values of angle $\gamma$ ( $2 / 3$ of measurements) ranged from $110^{\circ}$ to $140^{\circ}$, with values over $140^{\circ}$ constituting $15 \%$ of all measured values, and values under $100^{\circ}$ constituting $6 \%$ of measured values.

Alpha angle: Most angle $\alpha$ values (2/3 of all measurements) fell within the $130-160^{\circ}$ range, which 
A

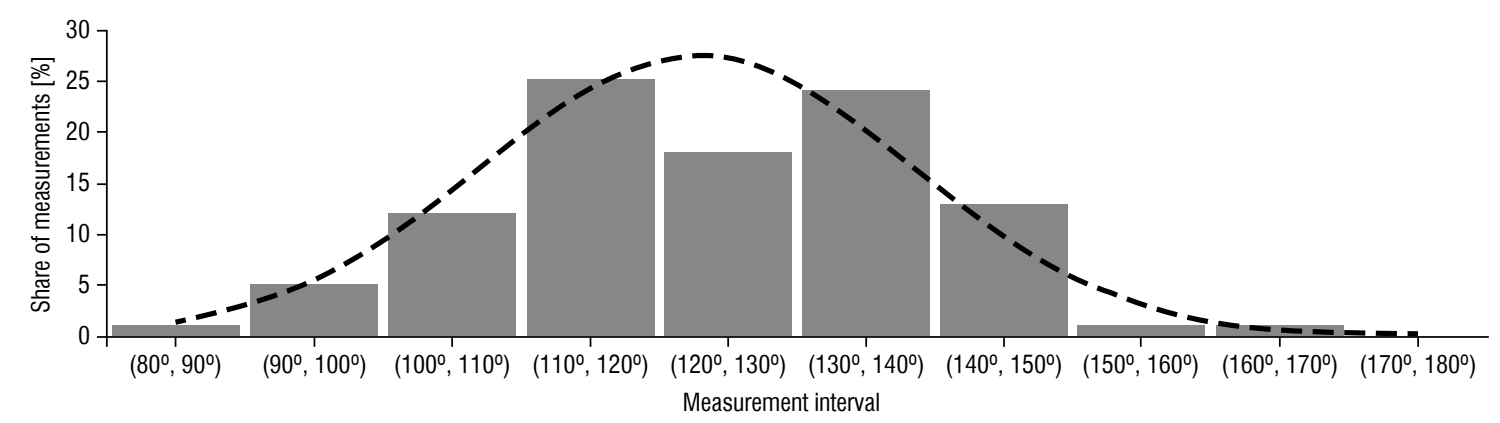

B

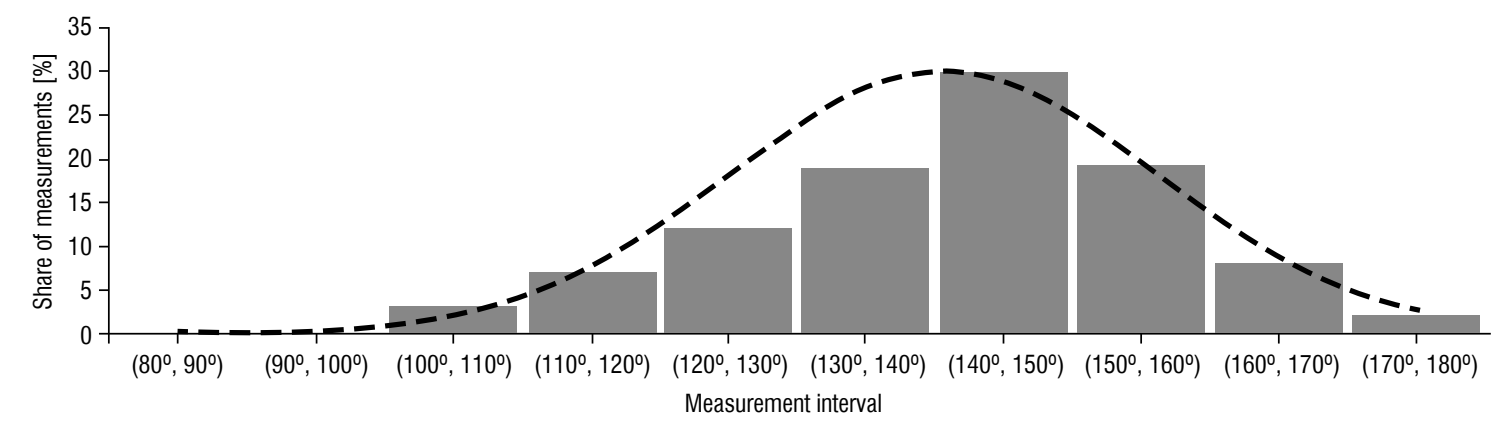

C

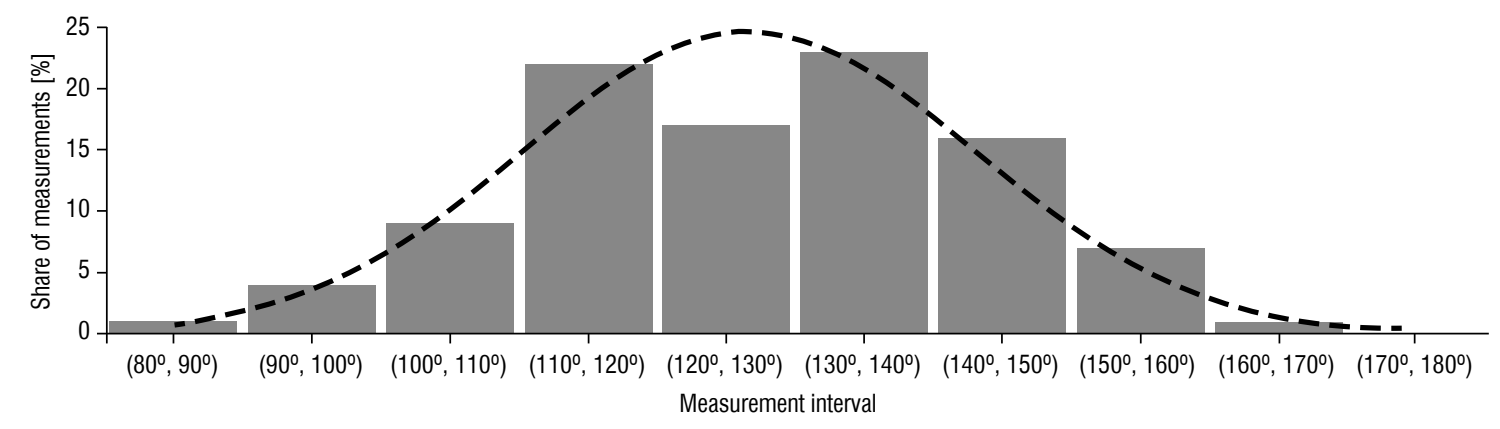

Figure 8. Histograms of the obtained values of angles gamma (A), alpha (B), and beta (C). The dashed lines mark the normal distributions estimated based on the obtained measurements.

made the angle, on average, more obtuse than either $\beta$ or $\gamma$. Values of over $160^{\circ}$ constituted $11 \%$ of the measurements, and no values under $100^{\circ}$ were observed.

Beta angle: The proportion of angles $\beta$ exceeding $140^{\circ}$ was $24 \%$ (which was greater than that of angles $\gamma$ ), with values under $100^{\circ}$ constituting $5 \%$ of the measurements.

The measurements obtained for all angles showed normal distributions (Shapiro-Wilk W test and Kolmogorov-Smirnov $D$ test) and homogeneity of variance (Leven's test), with the p-value of 0.05 .

\section{Comparisons between angles}

Comments: The scatter plots show a positive correlation between angles $\alpha$ and $\beta$ and angles $\alpha$ and $\gamma$.
Moreover, there is a slight positive correlation between angles $\gamma$ and $\beta$; however, it is less pronounced than the other two (Fig. 9A-C).

There was a significant, though weak, positive correlation between angles $\alpha$ and $\beta$ and angles $\alpha$ and $\gamma$. Higher values of angle $\alpha$ may be expected to be associated with higher mean values of angles $\gamma$ and $\beta$. The positive correlation between angles $\gamma$ and $\beta$ was not statistically significant; therefore, no conclusions can be drawn as to a correlation between these two angles.

Another confirmation of significant differences between the angles was provided by the results of analysis of variance and Student's $t$ test for the difference of means (with the Bonferonni correction due to multiple comparisons). 
A

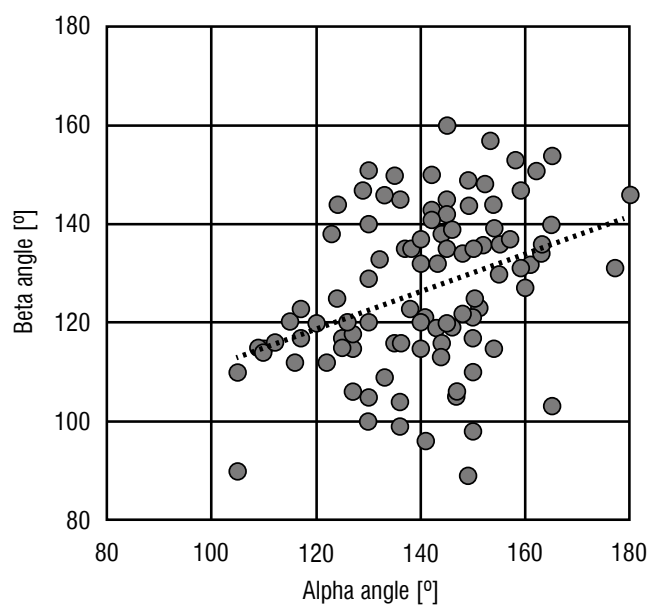

C

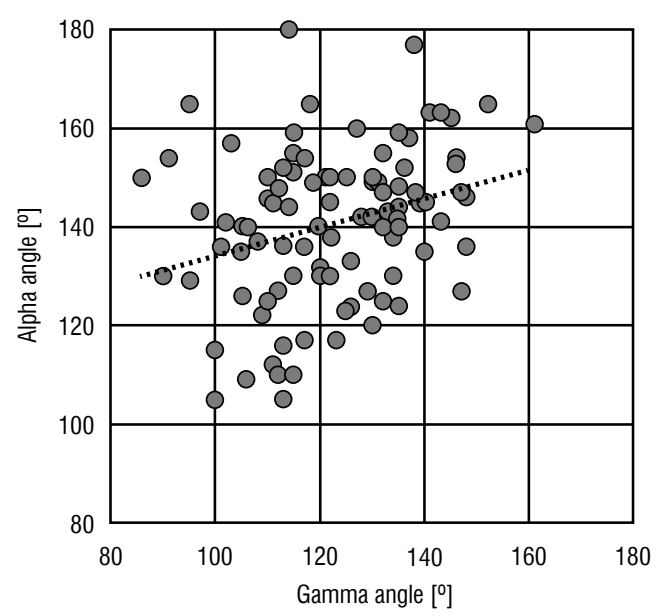

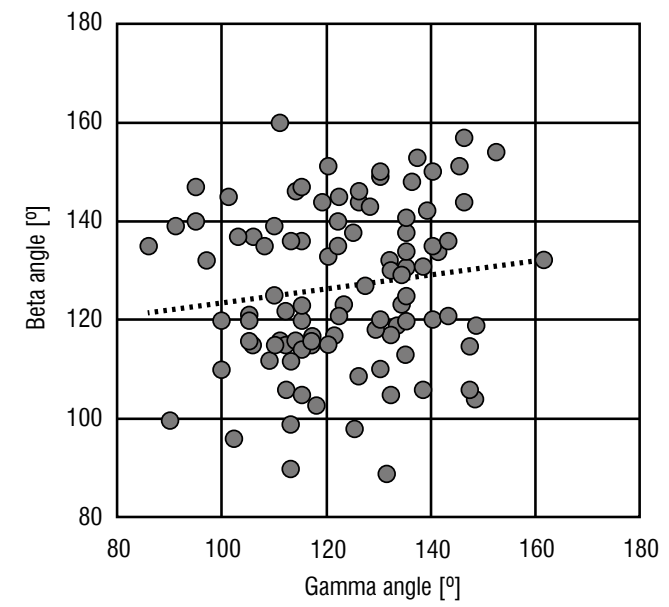

D

\begin{tabular}{|c|c|c|c|}
\hline & Alpha & Beta & Gamma \\
\hline Alpha & 1 & $\begin{array}{c}0.3673 \\
p=0.0002\end{array}$ & $\begin{array}{c}0.2942 \\
p=0.003\end{array}$ \\
\hline Beta & $\begin{array}{c}0.3673 \\
p=0.0002\end{array}$ & 1 & $\begin{array}{c}0.3673 \\
p=0.0002\end{array}$ \\
\hline Gamma & $\begin{array}{c}0.2942 \\
p=0.003\end{array}$ & $\begin{array}{c}0.1376 \\
p=0.1721\end{array}$ & 1 \\
\hline
\end{tabular}

Figure 9. A-C. Scatter graphs of the measurements for each pair of the evaluated angles. The slope of each dotted line of best fit shows the type of correlation; D. Pearson correlation coefficients between pairs of angles and their statistical significance.

Analysis of variance results demonstrated significant differences between the evaluated angles $(p<0.0001)$. The results of Student's $t$ test with $p=0.05$ showed significant differences between the mean angle values when comparing angles $\gamma$ and $\alpha$ and angles $\beta$ and $\alpha$, with no significant differences between angles $\gamma$ and $\beta$.

Therefore, we can conclude that angles $\alpha$ were significantly more obtuse than the other two types of analysed angles. However, we cannot state that angles $\gamma$ and $\beta$ differed significantly in their values.

\section{Drawing conclusions as to the value of a given angle based on the value of another angle}

Comments: Upper panel. Based on the value of angle $\gamma$ in the graph we can draw conclusions as to the likely values of angle $\alpha$. The graph shows, once again, a positive correlation between the values of angle $\alpha$ and those of angle $\gamma$ (this correlation is particularly clear for angle $\gamma$ values in the 100-170 range). Lower panel: The graph illustrates a positive correlation between the values of angles $\alpha$ and $\beta$, with higher values of angle $\alpha$ corresponding to higher expected values of angle $\beta$. This positive correlation is visible for the entire range of angle $\alpha$ values. There is no visible correlation between the values of angles $\gamma$ and $\beta$. Moreover, no such correlation was demonstrated in earlier analyses.

\section{DISCUSSION}

During normal human embryogenesis, the initially symmetrical venous system consists of two pairs of anterior and posterior cardinal veins, draining the cephalic and caudal areas of the embryo, respectively. These venous pairs anastomose to form the right and left common cardinal veins (ducts of Cuvier). At 


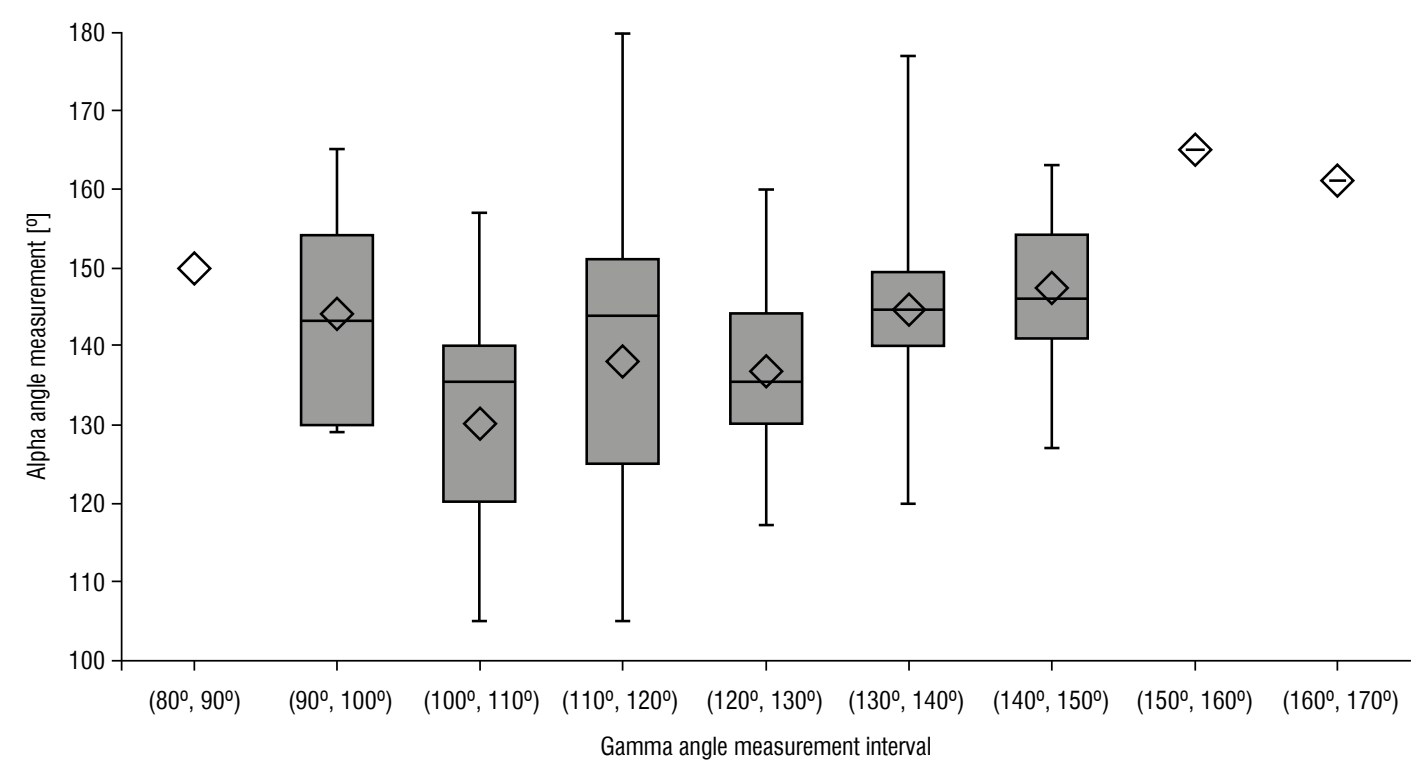

B

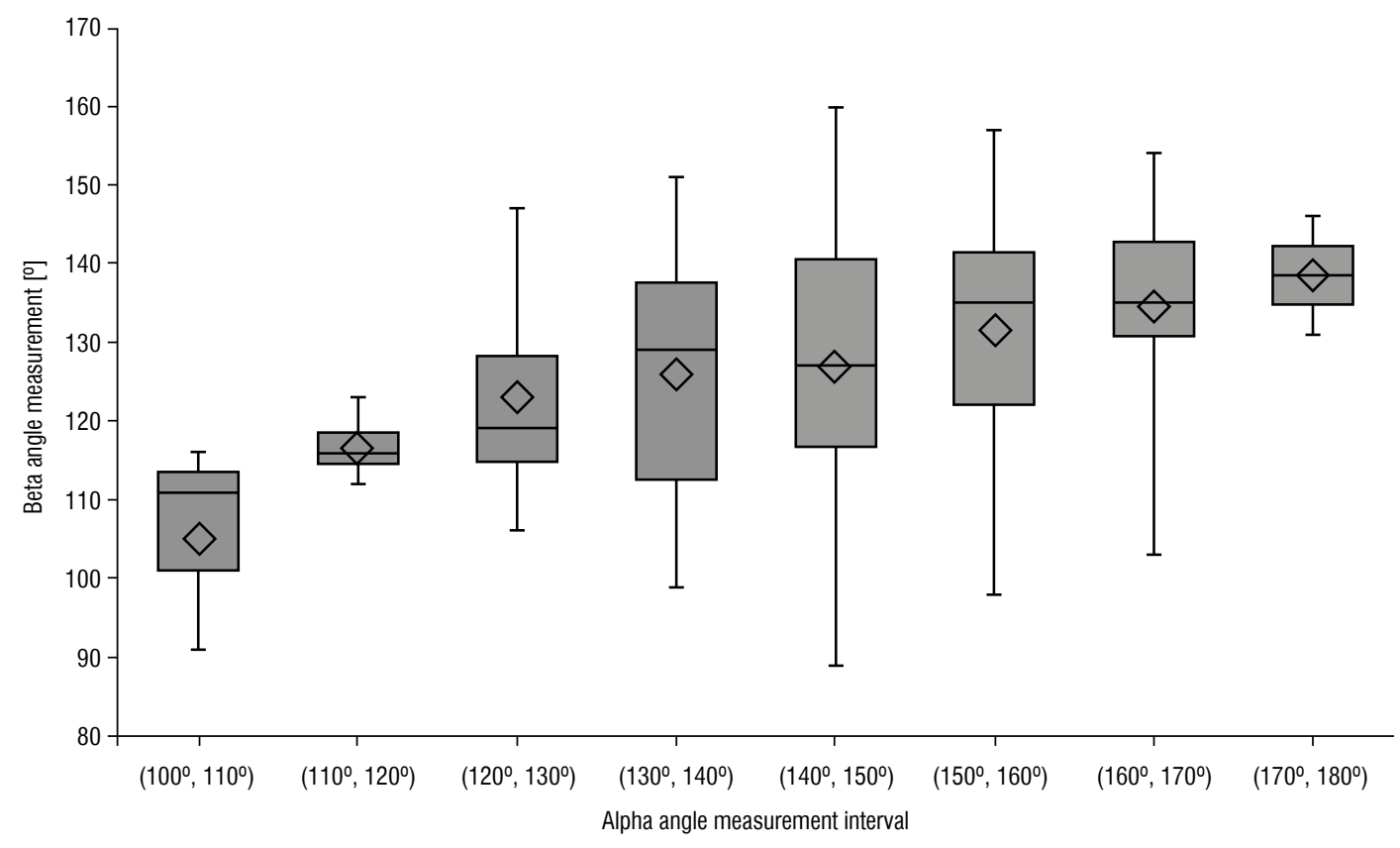

Figure 10. A, B. Box-and-whisker plots showing the ranges of angle measurements; A. The relationship between the value of angle $\alpha$ and the value of angle $\gamma ; \mathbf{B}$. The ranges of angle $\beta$ values with respect to the ranges of angle $\alpha$ values.

8 weeks of gestation, the left and right anterior cardinal veins coalesce to form BCVs. Eventually, the left $\mathrm{BCV}$ is nearly 3 times longer than its right counterpart [4]. Each of the BCVs drains its corresponding side of the chest by collecting blood from the ipsilateral internal jugular vein and SV as they join.

The most typical left BCV topography involves the vein coursing above the aortic arch and anterior to its branches (the brachiocephalic trunk, left common carotid artery, and left subclavian artery). Once it crosses to the right side of the upper mediastinum, the left $B C V$ joins the right $B C V$ to form the SVC [15].

During transvenous CIED implantation procedures, cardiac leads are typically introduced via the veins of the left clavipectoral triangle. In our centre this method is used in approximately $97 \%$ of de novo CIED implantation procedures. 


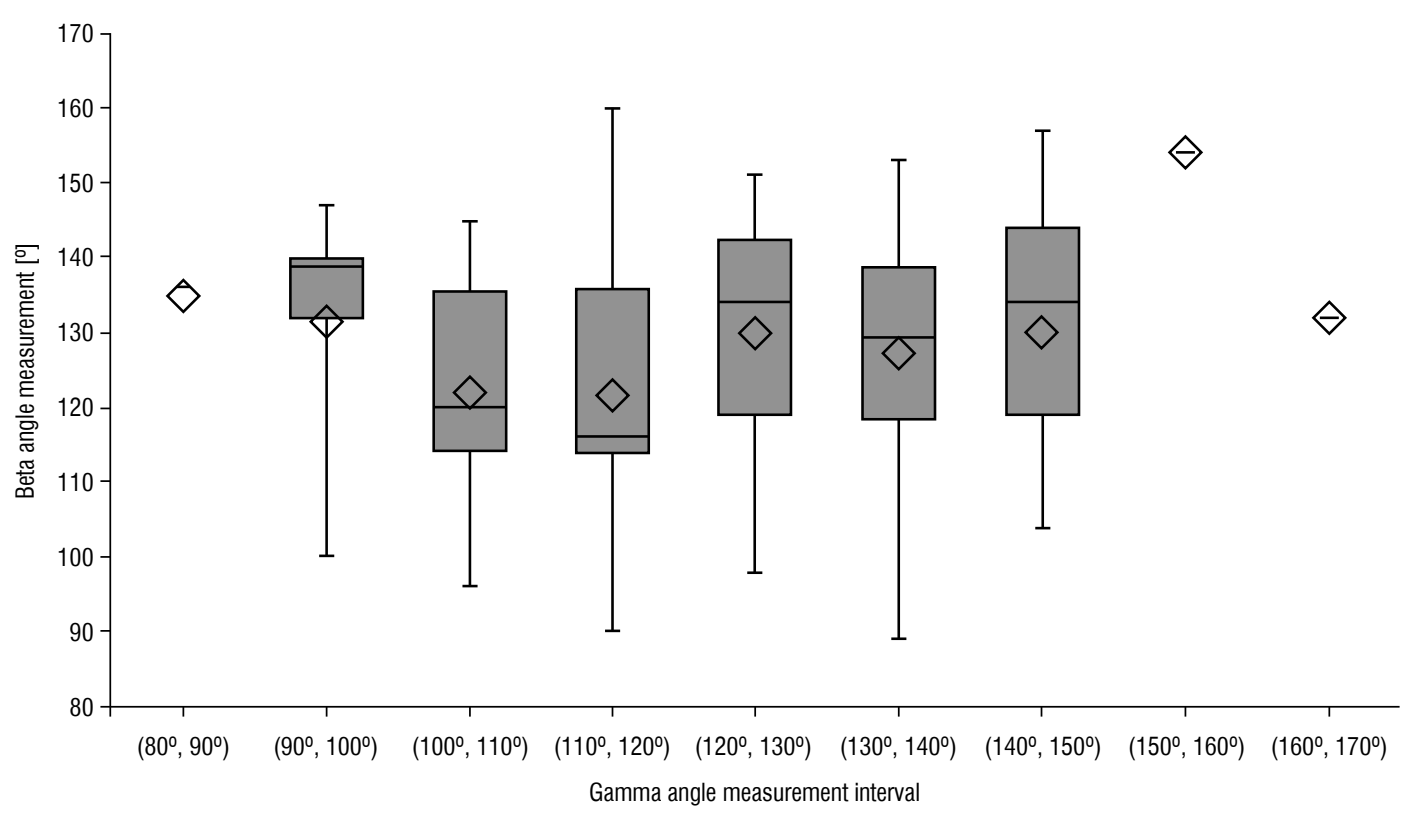

Figure 11. A box-and-whisker plot illustrating the distribution of angle $\beta$ values with respect to angle $\gamma$ value ranges.

Radiographic visualisation of the left BCV considerably helps in assessing the vein's morphometry and the nature of its angulations, as well as the position of these angulations with respect to adjacent structures. The nature of the angles along the course of the left BCV through the mediastinum helps observe any relationships between them (Figs. 9, 10). These relationships vary to some extent during chest movements during the expiratory phase of respiration (Fig. 1B, C).

The unique character of venous angulations may predispose to venous wall injuries during CIED implantation or CVC placement procedures. In severe cases, the injury may involve damage to adjacent anatomical structures, producing clinical manifestations. Nonetheless, even an epithelial injury may lead to long-term complications, such as post-thrombotic lesions $[1,9,18,21,22]$. The risk of vascular injury is increased by higher numbers of inserted leads, specific structural characteristics of the vein (diameter, wall stiffness, etc.), and any attempts to forcibly overcome potential difficulties in lead advancement $[6,8]$.

The risk of intraoperative venous injury is also increased in the vascular segments forming right-angle or sharp-angle angulations. In the analysed materials such vulnerable venous segments were: the site where the left SV and the left BCV meet (angle $\beta$ ) and the site of left BCV drainage to the SVC (angle $\gamma$ ) $[6,9,22]$. These angles created such unfavourable conditions in $5-6 \%$ of cases, collectively (Fig. 8A, B).
In most cases angle $\alpha$ was an obtuse angle (Fig. 8C), which facilitates lead advancement within the vessel. However, there is always a possibility of accidental lead advancement into the opening of such tributaries as the left superior intercostal vein or the left internal thoracic vein [20].

\section{Limitations of the study}

Our assessment of the rates and morphometric parameters of left BCV anatomical variations included a selected group of patients undergoing CIED implantation with the use of venography. Thus, the study population does not reflect the actual prevalence in the general population.

The angles of interest were measured based on contrast-enhanced images of left BCV lumina in posteroanterior views, without considering the potential lateral-view component of angle values.

The method of calculating the values of the angles marked graphically based on the intraluminal flow of the contrast agent may reflect some subjective bias; nonetheless, given the total number of measurements this does not seem to have significantly affected the conclusions, which are mostly based on relative differences.

\section{CONCLUSIONS}

The interindividual variations in left BCV topography and morphometry are largely due to the formation of the angulation in the middle of the vein's course through the mediastinum. 
Angles whose values were close to $90^{\circ}$ were found in $5 \%$ of cases and were most commonly at the site where the left SV continues into the left BCV and at the site where the left BCV drains into the SVC.

\section{Conflict of interest: None declared}

\section{REFERENCES}

1. Abu-El-Haija B, Bhave PD, Campbell DN, et al. Venous stenosis after transvenous lead placement: a study of outcomes and risk factors in 212 consecutive patients. J Am Heart Assoc. 2015; 4(8): e001878, doi: 10.1161/ JAHA.115.001878, indexed in Pubmed: 26231843.

2. Bowdle A. Vascular complications of central venous catheter placement: evidence-based methods for prevention and treatment. J Cardiothorac Vasc Anesth. 2014; 28(2): 358-368, doi: 10.1053/j.jvca.2013.02.027, indexed in Pubmed: 24008166.

3. Chen SJ, Liu KL, Chen HY, et al. Anomalous brachiocephalic vein: $\mathrm{CT}$, embryology, and clinical implications. AJR Am J Roentgenol. 2005; 184(4): 1235-1240, doi: 10.2214/ ajr.184.4.01841235, indexed in Pubmed: 15788602.

4. Chern MS, Ko JS, Tsai A, et al. Aberrant left brachiocephalic vein: $\mathrm{CT}$ imaging findings and embryologic correlation. Eur Radiol. 1999; 9(9): 1835-1839, doi: 10.1007/ s003300050931, indexed in Pubmed: 10602959.

5. Granata A, Zanoli L, Trezzi M, et al. Anatomical variations of the left anonymous trunk are associated with central venous catheter dysfunction. J Nephrol. 2018; 31(4): 571-576, doi: 10.1007/s40620-017-0465-z, indexed in Pubmed: 29270845.

6. Gravenstein N, Blackshear RH. In vitro evaluation of relative perforating potential of central venous catheters: comparison of materials, selected models, number of lumens, and angles of incidence to simulated membrane. J Clin Monit. 1991; 7(1): 1-6, doi: 10.1007/BF01617891, indexed in Pubmed: 1999693.

7. Guo X, Shi Y, Xie H, et al. Left innominate vein stenosis in an asymptomatic population: a retrospective analysis of 212 cases. Eur J Med Res. 2017; 22(1): 3, doi: 10.1186/ s40001-017-0243-3, indexed in Pubmed: 28115002.

8. Haghjoo M, Nikoo MH, Fazelifar AF, et al. Predictors of venous obstruction following pacemaker or implantable cardioverter-defibrillator implantation: a contrast venographic study on 100 patients admitted for generator change, lead revision, or device upgrade. Europace. 2007; 9(5): 328-332, doi: 10.1093/europace/eum019, indexed in Pubmed: 17369270.

9. Haq AA, Restrepo CS, Lamus D, et al. Thoracic venous injuries: an imaging and management overview. Emerg Radiol. 2016; 23(3): 291-301, doi: 10.1007/s10140-0161386-1, indexed in Pubmed: 26965007.

10. Bachleda JP. latrogenic injury to the superior vena cava and brachiocephalic vein. J Infect Dis Ther. 2014; 2(6): 169, doi: 10.4172/2332-0877.1000169.
11. Igawa O, Adachi M, Yano A, et al. Brachiocephalic vein perforation on three-dimensional computed tomography. Europace. 2007; 9(1): 74-75, doi: 10.1093/europace/ eul133, indexed in Pubmed: 17224430.

12. Kahkouee S, Sadr M, Pedarzadeh E, et al. Anomalous left brachiocephalic vein: important vascular anomaly concomitant with congenital anomalies and heart diseases. Folia Morphol. 2017; 76(1): 51-57, doi: 10.5603/ FM.a2016.0031, indexed in Pubmed: 27830886.

13. Ko SF, Huang CC, Ng SH, et al. Imaging of the brachiocephalic vein. AJR Am J Roentgenol. 2008; 191(3): 897-907, doi: 10.2214/AJR.07.3552, indexed in Pubmed: 18716126.

14. Ko SF, Ng SH, Fang FM, et al. Left brachiocephalic vein perforation: computed tomographic features and treatment considerations. Am J Emerg Med. 2007; 25(9): 1051-1056, doi: 10.1016/j.ajem.2007.06.013, indexed in Pubmed: 18022501.

15. Mitsuoka H, Arima T, Ohmichi $Y$, et al. Analysis of the positional relationship between the left brachiocephalic vein and its surrounding vessels via computed tomography scan: A retrospective study. Phlebology. 2020; 35(6): 416-423, doi: 10.1177/0268355519898320, indexed in Pubmed: 31918641.

16. Nakabayashi K. latrogenic brachiocephalic vein perforation during pacemaker implantation. BMJ Case Rep. 2015; 2015(29), doi: 10.1136/bcr-2015-209369, indexed in Pubmed: 25634862.

17. Rozmus G, Daubert JP, Huang DT, et al. Venous thrombosis and stenosis after implantation of pacemakers and defibrillators. J Interv Card Electrophysiol. 2005; 13(1): 9-19, doi: 10.1007/s10840-005-1140-1, indexed in Pubmed: 15976973.

18. Salik E, Daftary A, Tal MG. Three-dimensional anatomy of the left central veins: implications for dialysis catheter placement. J Vasc Interv Radiol. 2007; 18(3): 361-364, doi: 10.1016/j.jvir.2006.12.721, indexed in Pubmed: 17377181.

19. Steckiewicz R, Kosior DA, Rosiak M, et al. The prevalence of superior vena cava anomalies as detected in cardiac implantable electronic device recipients at a tertiary cardiology centre over a 12-year period. Hellenic J Cardiol. 2016; 57(2): 101-106, doi: 10.1016/j.hjc.2016.03.003, indexed in Pubmed: 27445024.

20. Steckiewicz R, Stolarz P, Świętoń E, et al. Tributaries of the left brachiocephalic vein posing problems in central venous catheterization and, potentially, in cardiac implantable electronic device placement. Folia Cardiologica. 2020; 15(4): 277-284, doi: 10.5603/fc.a2020.0040.

21. Teichgräber UK, Gebauer B, Benter T, et al. Central venous access catheters: radiological management of complications. Cardiovasc Intervent Radiol. 2003; 26(4): 321-333, doi: 10.1007/s00270-003-0112-z, indexed in Pubmed: 14667113.

22. Vertemati M, Rizzetto F, Cassin S, et al. Clinical relevance of the left brachiocephalic vein anatomy for vascular access in dialysis patients. Clin Anat. 2020; 33(8): 1120-1129, doi: 10.1002/ca.23549, indexed in Pubmed: 31891199. 